OPEN ACCESS

Edited by:

Yogen Singh,

Cambridge University Hospitals NHS

Foundation Trust, United Kingdom

Reviewed by:

Lizelle Van Wyk

Stellenbosch University, South Africa

Alison J. Carey,

Drexel University, United States

${ }^{*}$ Correspondence:

Eleanor J. Molloy

eleanor.molloy@tcd.ie

Specialty section:

This article was submitted to

Neonatology

a section of the journal

Frontiers in Pediatrics

Received: 25 August 2020 Accepted: 24 December 2020 Published: 15 February 2021

Citation:

O'Dea MI, Kelly L, McKenna E,

Melo AM, Ni Bhroin M, Hurley T, Byrne AT, Colleran G, Vavasseur C, El-Khuffash A, Miletin J, Murphy J,

Hickey F and Molloy EJ (2021)

Dysregulated Monocyte and Neutrophil Functional Phenotype in Infants With Neonatal Encephalopathy Requiring Therapeutic Hypothermia.

Front. Pediatr. 8:598724.

doi: 10.3389/fped.2020.598724

\section{Dysregulated Monocyte and Neutrophil Functional Phenotype in Infants With Neonatal Encephalopathy Requiring Therapeutic Hypothermia}

\author{
Mary Isabel O'Dea 1,2,3, Lynne Kelly', Ellen McKenna ${ }^{2}$, Ashanty M. Melo'2, \\ Megan Ni Bhroin ${ }^{4}$, Tim Hurley ${ }^{5}$, Angela T. Byrne ${ }^{6}$, Gabrielle Colleran ${ }^{7}$, \\ Claudine Vavasseur ${ }^{8}$, Afif El-Khuffash ${ }^{9}$, Jan Miletin ${ }^{1}$, John Murphy ${ }^{8}$, Fionnuala Hickey ${ }^{10}$ \\ and Eleanor J. Molloy 1,2,3,11,12* \\ ${ }^{1}$ Department of Paediatrics and Neonatology, Coombe Women \& Infants University Hospital, Dublin, Ireland, ${ }^{2}$ Trinity \\ Translational Medicine Institute, Trinity College Dublin, Dublin, Ireland, ${ }^{3}$ National Children's Research Centre (NCRC), Crumlin, \\ Ireland, ${ }^{4}$ Trinity College Institute of Neuroscience and Cognitive Systems Group, Discipline of Psychiatry, School of Medicine, \\ Trinity College Dublin, Dublin, Ireland, ${ }^{5}$ Department of Paediatrics and Child Health, Trinity College Dublin, Dublin, Ireland, \\ ${ }^{6}$ Children's Health Ireland at Crumlin, Dublin, Ireland, ${ }^{7}$ Department of Radiology, National Maternity Hospital, Dublin, Ireland, \\ ${ }^{8}$ National Maternity Hospital, Dublin, Ireland, ${ }^{9}$ Rotunda Hospital, Dublin, Ireland, ${ }^{10}$ Trinity Health Kidney Centre, Faculty of \\ Health Sciences, School of Medicine, Trinity College Dublin, Dublin, Ireland, ${ }^{11}$ Our Lady's Children's Hospital (CHI), Crumlin, \\ Ireland, ${ }^{12}$ Department of Paediatrics, Tallaght University Hospital, Dublin, Ireland
}

Neonatal encephalopathy (NE) is a significant cause of morbidity and mortality. Persistent inflammation and activation of leukocytes mediate brain injury in NE. The standard of care for NE, therapeutic hypothermia $(\mathrm{TH})$, does not improve outcomes in nearly half of moderate to severe cases, resulting in the need for new adjuvant therapies, and immunomodulation holds promise. Our objective was to explore systemic leukocyte phenotype in infants with $\mathrm{NE}$ and healthy controls in response to lipopolysaccharide (LPS). Twenty-four infants with NE (NE II-20; NE III =4) requiring $\mathrm{TH}$ and 17 term neonatal controls were enrolled, and blood samples were analyzed between days 1 and 4 of life at a mean (SD) timepoint of 2.1 ( \pm 0.81) days of postnatal life at the time of the routine phlebotomy. Leukocyte cell surface expression levels of Toll-like receptor 4, NADPH oxidase (NOX2), CD11b, mitochondrial mass, and mitochondrial superoxide production were measured by flow cytometry. Gene expression of TRIF (TIR domain-containing adapter-inducing interferon- $\beta$ ), MyD88 and IRAK4 was measured by reverse transcription-polymerase chain reaction. Infants with NE had significantly lower expression of neutrophil CD11b and NOX2 with LPS stimulation compared to healthy term controls. Mitochondrial mass in neutrophils and monocytes was significantly increased in NE infants with LPS compared to controls, potentially indicating a dysregulated metabolism. Infants with NE had significantly lower IRAK4 at baseline than controls. NE infants display a dysregulated inflammatory response compared to healthy infants, with LPS hyporesponsiveness to CD11b and NOX2 and decreased IRAK4 gene expression. This dysregulated immune profile may indicate an adaptable response to limit hyperinflammation.

Keywords: neuroimmunology, monocytes, neutrophils, neonatal encephalopathy, innate immunity 


\section{INTRODUCTION}

The neonatal period is the most vulnerable time for childhood mortality, $44 \%$ of deaths in the population younger than 5 years occur during this time, with $29 \%$ of these attributed to neonatal encephalopathy (NE) (1). The estimated global burden of NE is 1.15 million, with $96 \%$ of NE infants born in low- and middleincome countries (2). There has been significant progress in the management of NE with the development of therapeutic hypothermia (TH) as the standard of care; however, morbidity and mortality remain high (3).

Immune dysfunction is well-described in NE; nevertheless, prior to developing new immunomodulatory therapies, further understanding of the inflammatory phenotype is crucial $(4,5)$. For instance, altered levels of proinflammatory and antiinflammatory cytokines and a dysregulated cellular inflammatory response to endotoxin have been demonstrated in $\mathrm{NE}(1,2,6)$. Different immunomodulators have been postulated to target inflammation, excitotoxicity, and oxidative stress in NE, such as the nucleotide-binding domain-like receptor protein 3 (NLRP3) inflammasome (7).

Neutrophils and monocytes are involved in systemic inflammation in NE (1). Toll-like receptor 4 (TLR-4) is an immune cell surface receptor involved in recognizing endotoxin, and CD11b is a marker of cell activation. Myeloid differentiation primary response (MyD88) and TIR domain-containing adapter-inducing interferon- $\beta$ (TRIF) are adaptors that bind to the intracellular domains of the TLR and interleukin-1 receptor families, linking them to IL-1R-associated kinase (IRAK) (8). Activation of IRAK activates downstream inflammatory pathways leading to expression of proinflammatory cytokines: tumor necrosis factor $\alpha$, interleukin $1 \beta$ (IL-1 $\beta$ ), IL-6, and interferon $\gamma(8)$.

Mitochondria are a source of reactive oxygen species (ROS) in the cell; in many pathologies, ROS contributes to mitochondrial damage (9). Glycolysis and oxidative phosphorylation support monocyte function; during inflammation, the mitochondria allows macrophages to switch from glycolysis to oxidative phosphorylation (9). Neutrophil mitochondria depend on anaerobic glycolysis and are adapted to working under hypoxia (10).

We hypothesized that infants with NE may have an altered inflammatory phenotype in terms of markers of activation CD11b, TLR-4 [lipopolysaccharide (LPS) responsiveness], and NOX2. We examined underlying mechanisms of the immunophenotype in NE at the mRNA level for TLR-4 signaling and examined the mechanistic role of the mitochondria in neutrophils and monocytes by examining superoxide production and mitochondrial mass.

\footnotetext{
Abbreviations: IL, Interleukin; IRAK, IL-1R-associated kinase; LPS, Lipopolysaccharide; MFI, Mean fluorescence intensity; MTG, MitoTracker green; MyD88, Myeloid differentiation primary response; NADPH, Nicotinamide adenine dinucleotide phosphate; NE, Neonatal encephalopathy; NLRP3, Nucleotide-binding domain-like receptor protein 3; NOX2, NADPH oxidase; ROS, Reactive oxygen species; TH, Therapeutic hypothermia; TLR-4, Toll-like receptor 4; TRIF, TIR domain-containing adapter-inducing interferon- $\beta$.
}

\section{METHODS}

\section{Study Population}

This study was approved by the ethics committees of the three Dublin Maternity Hospitals, which are all tertiary neonatal intensive care units (NICUs) and national referral centers for TH. Families received verbal and documented information on the study, and written consent was obtained prior to recruitment.

We have previously recruited several distinct cohorts of infants with NE and controls with criteria as described $(2,11)$. The NE severity was classified by Sarnat staging (12). Infants with NE had a magnetic resonance imaging (MRI) of the brain completed and scored as per Barkovich classification, a scoring system for the assessment of brain injury that is predictive of motor outcome. The score evaluates basal ganglia injury, watershed injury, combined injury score, and summation score (13). Infants with congenital abnormalities or evidence of maternal substance abuse were excluded. Asymptomatic wellterm infants undergoing routine phlebotomy were included as controls, but infants undergoing sepsis evaluations or receiving phototherapy for jaundice were excluded.

\section{Blood Sampling}

Whole-blood sampling was performed following informed parental consent. Sampling was performed using aseptic technique via central and peripheral arterial lines and via venous sampling at times of routine patient phlebotomy at one timepoint during their NICU course. The blood was collected in sodium citrate tubes and processed immediately. The volume taken was 1-1.4 mL, which was approved by the ethics committee, as a volume that would not affect the infant's hemodynamic status.

\section{Cell Surface Antigen Expression}

The expression of TLR4, NOX2, and CD11b on the surface of neutrophils and monocytes was evaluated by flow cytometry. Whole blood $(200 \mu \mathrm{L})$ was treated in $100-\mu \mathrm{L}$ aliquots incubated at $37^{\circ} \mathrm{C}$ for $1 \mathrm{~h}$ untreated (vehicle) or with $10 \mathrm{ng} / \mathrm{mL}$ LPS (SIGMA Life Science, Ireland). Fluorochrome-conjugated monoclonal antibodies (mAb) specific for humans CD14-PerCP, CD15-PECy7, NOX2-FITC, CD66b-Pacific Blue, TLR4-APC (BioLegend ${ }^{\circledR}$, USA), and CD11b-PE (BD Biosciences, UK) were used. The whole blood was then stained with $\mathrm{mAb}$ for $15 \mathrm{~min}$. Red blood cells were lysed with BD lysis buffer. Cells were acquired on a FACSCanto II flow cytometer (BD Bioscience) and analyzed using FlowJo version 10 (Tree Star) $(2,14)$. Neutrophils were delineated based on SSC-A and $\mathrm{CD}_{6} 6 \mathrm{~b}^{+}$and monocytes based on SSC-A, CD66b ${ }^{-}$, and $\mathrm{CD}_{14}^{+}(15,16)$. Monocytes were subdivided into classical, intermediate, and non-classical subtypes. Monocyte subsets were identified as classical: CD $14^{\text {high }} \mathrm{CD} 16^{\text {neg/low }} \quad$ intermediate: $\quad \mathrm{CD} 14^{\text {high }} \mathrm{CD} 16^{\text {high }}$; non-classical: $\mathrm{CD} 14^{\text {low }} \mathrm{CD} 16^{\text {high }}$. A minimum of 10,000 events were collected, and relative expression of antigens was expressed as mean fluorescence intensity (MFI).

\section{Mitochondrial Mass and ROS}

MitoTracker green (MTG) was used to assess mitochondrial mass of leukocytes. MitoSox red was used to assess mitochondrial superoxide production of leukocytes. Generation 
of mitochondrial ROS (mtROS) and mitochondrial mass were evaluated using flow cytometry. Whole blood was incubated at $37^{\circ} \mathrm{C}$ for $1 \mathrm{~h}$ untreated (vehicle) or with $10 \mathrm{ng} / \mathrm{mL}$ LPS in the presence of MTG dye $(1.4 \mu \mathrm{M})$ and MitoSox red reagent $(15 \mu \mathrm{M})$ (ThermoFisher Scientific). Samples were stained with $\mathrm{mAb}$ for CD15- PECy7, CD66b-PB (BioLegend ${ }^{\circledR}$, USA), CD14-APC (Beckman Coulter), and CD16 ${ }^{-}$V500 (BD Biosciences, UK) for $15 \mathrm{~min}$. BD lysis buffer was used to lyse red blood cells. Cells were acquired on a BD FACSCanto II flow cytometer. Fluorescence minus one (FMO) controls were used to set gates. Mitochondrial mass and superoxide were quantified based on MFI of MTG and MitoSOX red, respectively (17). Data were analyzed using FlowJo software version 10.

\section{Reverse Transcription-Polymerase Chain Reaction}

Gene expression of TRIF, IRAK4, and MyD88 in whole blood in the presence or absence of LPS was examined using reverse transcription-polymerase chain reaction (RT-PCR). Following whole-blood incubation of samples with LPS, RNA extraction was performed using Ribopure blood kit as per manufacturer (ThermoFisher). RNA purity and concentration were measured using a NanoDrop ND-100 spectrophotometer and analyzed using ND-1000 version 3.1.2 software. Total RNA, $1 \mu \mathrm{g}$, was reverse transcribed to single-stranded cDNA using the High-Capacity cDNA Archive Kit (Applied Biosystems) as per manufacturer. The settings for amplification were $10 \mathrm{~min}$ at $25^{\circ} \mathrm{C}, 120 \mathrm{~min}$ at $37^{\circ} \mathrm{C}$, and $5 \mathrm{~s}$ at $85^{\circ} \mathrm{C}$ and holding at $4^{\circ} \mathrm{C}$. TaqMan ${ }^{\circledR}$ primer and probe combinations were used to detect gene expression of MyD88 (NM_001172567.1), TRIF (NM_182919.3), and IRAK4 (NM_001114182.2). Thermal cycling conditions were as follows: $2 \mathrm{~min}$ at $50^{\circ} \mathrm{C}, 10 \mathrm{~min}$ at $95^{\circ} \mathrm{C}$, and, for 40 cycles, $24 \mathrm{~s}$ at $95^{\circ} \mathrm{C}$ and $1 \mathrm{~min}$ at $60^{\circ} \mathrm{C}$, using a $7900 \mathrm{HT}$ Fast Real-Time PCR System. Relative quantification values were calculated using the $2^{-\Delta \Delta \mathrm{Ct}}$ method (16).

\section{Statistics}

Statistical analysis was performed on GraphPad PRISM version 8 using unpaired (unmatched) or paired (matched), two-tailed $t$-tests to compare mean results between two independent cohorts. The Kolmogorov-Smirnov test was used to check normality. Significance was defined as $p<0.05$. Results shown are expressed as mean \pm standard error of the mean.

\section{RESULTS}

\section{Patient Demographics}

Twenty-four infants with NE (NE II, $n=20$; NE III, $n=4$ ) who required $\mathrm{TH}$ and 17 term neonatal controls were enrolled. The infants with NE had their phlebotomy performed at a mean (SD) timepoint of 2.1 ( \pm 0.81$)$ days of postnatal life, on day $1(n=5)$, day $2(n=14)$, day $3(n=3)$, and day $4(n=2)$.

In the NE group, the mean (SD) Apgar scores were 2.9 (2.0) and 5.0 (2.3) at 1 and $5 \mathrm{~min}$, respectively, and no infants died. The mean (SD) birth weight was $3.4(0.5) \mathrm{kg}$, and there were 16 male infants. Infants with NE had an MRI brain, assessed with the Barkovich scoring classification as follows: eight infants had a normal scan, eight infants had isolated watershed injury, and no infants had an isolated basal ganglia injury. Seven infants had both watershed and basal ganglia injury. Two infants did not have an MRI Barkovich score completed.

The term control infants had a mean (SD) gestation of 39.2 (1.6) weeks, birth weight of $3.3(0.5) \mathrm{kg}$, and Apgar scores of 9 and $10( \pm 0)$ at 5 and $10 \mathrm{~min}$, and the group included five male infants.

\section{Leukocyte Cell Surface Markers}

A significant increase in neutrophil CD11b expression following LPS stimulation was observed in controls $(p=0.021)$ but not in NE infants (Figure 1A). No difference in monocyte CD11b expression between healthy controls and infants with NE was observed (Figure 1B). Neutrophil TLR4 was lower in infants with NE at baseline compared to controls and following LPS stimulation ( $p=0.05$ ) (Figure 1C). Significantly elevated neutrophil NOX2 expression with LPS was demonstrated in controls but not in NE infants ( $p=0.046)$ (Figure 1E).

Infants with NE showed relative LPS hyporesponsiveness to neutrophil CD11b and NOX2 expression indicating an altered inflammatory response.

\section{Mitochondrial Mass and mtROS}

Following treatment with LPS, neutrophils $(p=0.002$, Figure 2A) and monocytes $(p=0.038$. Figure 2B) from infants with NE had significantly higher mitochondrial mass in comparison with healthy term controls. Following treatment with LPS, all monocyte subsets (Figures 2C-E) from infants with $\mathrm{NE}$ had significantly higher mitochondrial mass in comparison with controls (all $p<0.05$ ).

Infants with NE displayed higher mitochondrial mass than controls in response to LPS, which may potentially demonstrate that infants with NE have higher levels of metabolism and superoxide production than healthy controls.

There were no significant differences in mtROS production for neutrophils or monocytes between controls and infants with NE (Figure 3).

\section{TRIF, IRAK4, and MyD88}

There was significantly lower IRAK4 expression in NE infants at baseline than control infants $(p=0.014)$ with no differences detected upon LPS stimulation (Figure 4A). No difference in TRIF or MyD88 expression between infants with NE and controls was observed (Figures 4B,C). Lower IRAK4 gene expression in NE infants compared to control infants suggests a possible IRAK4 deficiency in NE infants.

\section{DISCUSSION}

We found that infants with NE undergoing $\mathrm{TH}$ displayed neutrophil and monocyte LPS hyporesponsiveness for surface expression of NOX2 and CD11b. IRAK4 gene expression was lower in NE at baseline, whereas mitochondrial mass was higher in NE with LPS compared to controls.

CD11b was significantly increased on the surface of neutrophils in healthy controls but not in infants with NE. 


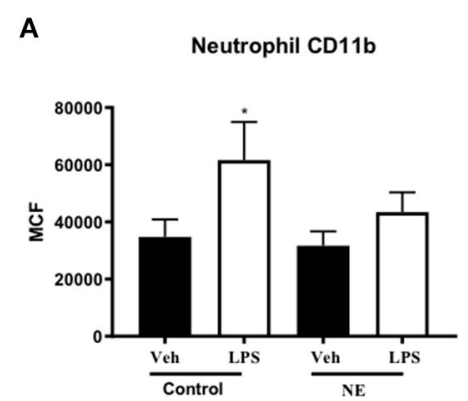

B

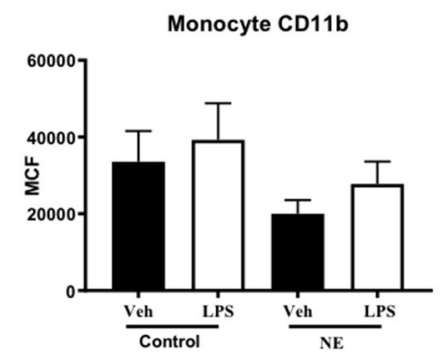

c

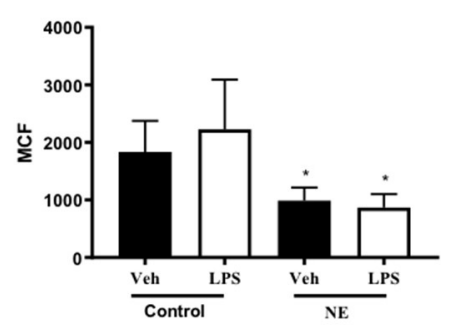

D

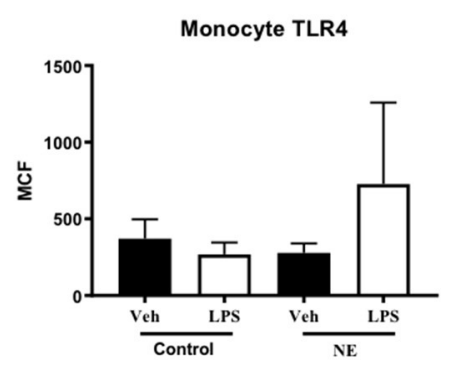

E

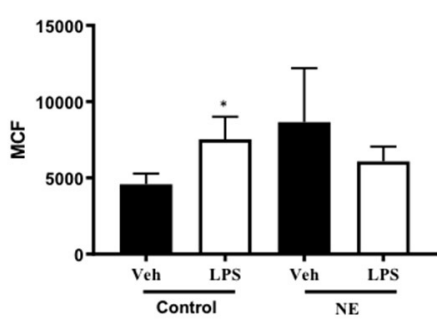

$\mathbf{F}$



FIGURE 1 | CD11b, TLR-4, and NOX2 expression in healthy controls and in neonatal encephalopathy. Whole blood from NE patients and controls was stimulated with LPS and leukocyte markers were analyzed by flow cytometry. Graph shows MCF of CD11b, TLR-4, and NOX2 on neutrophils and monocytes. Neutrophils and monocytes were identified based on their size and granularity (FSC, forward scatter; and SSC, side scatter, respectively), followed by labeling with CD66 ${ }^{+}$for granulocytes, and CD66 $b^{-}$and CD14+ for monocytes. Results are expressed as mean channel fluorescence (MCF); "veh" refers to unstimulated ( ${ }^{*} p<0.05$ using unpaired, two-tailed t-test). A significant increase in neutrophil CD11b expression following LPS stimulation was observed in controls ( $p=0.021)$ but not in NE infants (A). Neutrophil TLR4 was lower in infants with NE at baseline compared to controls and following LPS stimulation ( $p=0.05)$ (C). Significantly elevated neutrophil NOX2 expression with LPS was demonstrated in controls but not in NE infants ( $p=0.046)$ (E). Monocyte Cd11b, TLR4, and Nox expression did not differ between controls and in NE at baseline nor with LPS stimulation (B,D,F).

Neutrophils from infants with NE II/III had decreased CD11b in response to LPS compared to controls (18). Neutrophils are the earliest leukocyte to initiate the inflammatory response; hypoxia can delay neutrophil apoptosis, causing a persistent inflammatory response, increased ROS production, and CD11b activation (18). O'Hare et al. demonstrated higher monocyte and neutrophil CD11b in NE infants compared to adult blood at baseline and in response to LPS stimulation but lacked neonatal controls; hence, the comparator group was not as clinically relevant as our own study with neonatal controls (1). The different comparator group makes it difficult to draw significant comparisons between the studies. Children in pediatric intensive care had decreased leukocyte CD11b LPS responses compared to healthy adult and pediatric controls, correlating with our findings (19).

NOX2 expression was significantly elevated with LPS in control infants' neutrophils but not in NE neutrophils (Figure 1). NADPH oxidase increases upon hypoxia-ischemia and contributes to brain injury by production of ROS (10). Following LPS stimulation ex vivo, neonates with severe brain injury produced significantly higher systemic neutrophil ROS compared to mildly affected neonates (NE 0/I) at $72-96 \mathrm{~h}$ and day 7 of life, but the study lacked neonatal controls (20). Our data may suggest a potential neuroprotective role of NOX2 in healthy infants, and a lack of increase in NE neutrophil NOX2 may suggest these infants cannot mount a protective response.

Neutrophil TLR-4 surface expression was lower in NE than control at baseline and after LPS stimulation. TLR-4 is upregulated with endotoxin in brain injury in vivo (14). TLR-4 knockout mice have lower infarct size in stroke model compared to wild type, suggesting that antagonizing TLR-4 may have a role in neuroprotection (21).

mtROS production via neutrophils and monocytes was similar in infants with NE and controls at baseline and with LPS stimulation. ROS can cause significant tissue damage and may be mediated by NADPH oxidase, mitochondrial dysfunction, and hypoxia-inducible factor $1 \alpha$ upregulation. Mitochondria are vulnerable in infants with $\mathrm{NE}$ as their capacity to scavenge is overcome by high levels of ROS, initiating mitochondrial permeability transition and oxidative stress (22). Neutrophil mtROS production increased in pediatric patients with systemic lupus erythematosus compared to healthy pediatric controls (23). Mitochondrial autophagy, the ability of mitochondria to maintain homeostasis and adapt to stress, may explain the relatively few differences in NE and controls (24). However, the predominant form of ROS in neutrophils is produced by 




C

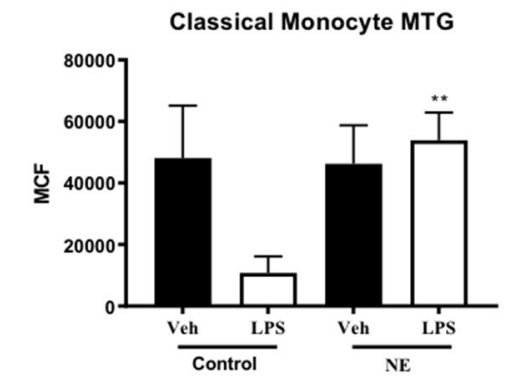

B

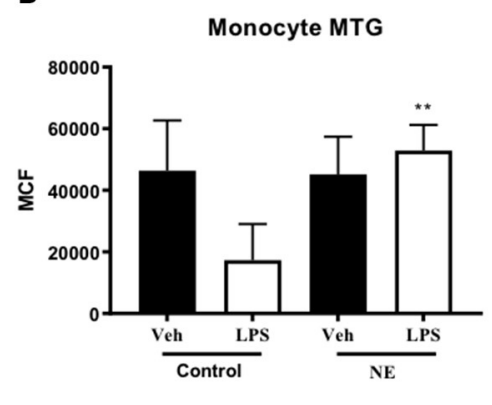

D

Intermediate Monocyte MTG

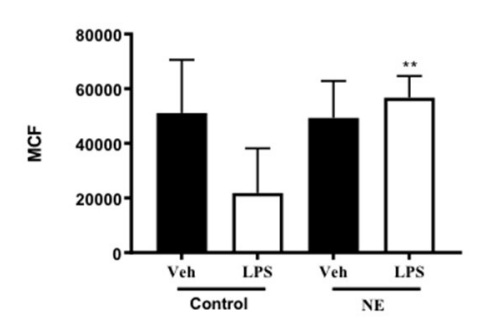

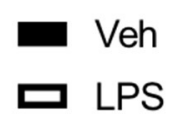

E

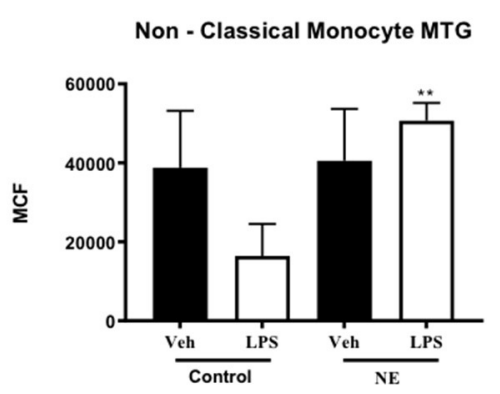

FIGURE 2 | Neutrophil and monocyte mitochondrial mass in healthy term controls compared to NE. MitoTracker (MTG) green was used to measure mitochondrial mass of leukocytes in whole blood from NE patients and controls following LPS stimulation. Graphs show MTG of (A) neutrophils, (B) monocytes, (C) classical monocyte MTG, (D) intermediate monocyte MTG, (E) non-classical monocyte MTG, in healthy term controls $(n=4-5)$ and NE infants $(n=4-5)$. CD14 and CD16 FMO ensured a standardized way of dividing monocytes into distinct populations in the context of the data spread due to the multiple fluorochromes in the panel. Monocytes were divided into classical, non-classical, and intermediate monocytes. Results are expressed as mean channel fluorescence (MCF); "veh" refers to unstimulated $\left({ }^{*} p<0.05,{ }^{\star *} p<0.01\right.$ using unpaired two-tailed $t$-test).

NADPH oxidase; not mitochondria (20) but mtROS are involved in NOX2 activation. Exocytosis of both primary and secondary granules, and delayed apoptosis and contributes to excessive activation of neutrophils (25). We have previously shown that neonates with abnormal neuroimaging and/or severe NE had increased neutrophil total ROS as well as CD11b and TLR-4 expression (1). An augmented neutrophil total ROS response in infants with NE post-LPS compared to neonatal controls was reduced significantly by activated protein C (26). In addition, although there is similar neutrophil ROS production in preterm and term neonates with high levels of total ROS in the neonatal controls (27). Neutrophils and monocytes of NE infants showed a significantly increased mitochondrial mass than controls with LPS. Endotoxin sensitizes the immature brain to damage in animal models, and infants exposed to in utero inflammation by infection have a higher risk of NE (28). Metabolic dysfunction is a hallmark of NE. In the immature brain, mitochondrial swelling results from excessive calcium influx leading to increased superoxide production (28). Elevated mitochondrial mass of leukocytes may be indicative of brain injury typical of NE.

IRAK4 expression was significantly lower in NE than in controls at baseline but did not change with LPS stimulation. There were no differences in TRIF and MyD88 between NE and controls. MyD88, TRIF, and IRAK are adaptor molecules that activate downstream inflammatory responses following TLR activation (8). IRAK inhibition is protective in a necrotizing enterocolitis animal model by preventing the release of proinflammatory cytokines and reducing intestinal damage (29). IRAK4 deficiency in humans impairs TLR innate immunity and causes increased susceptibility to bacterial infections including Staphylococcus aureus and Streptococcus pneumoniae (30). In most patients with IRAK4 deficiency, the first infection presents before 2 years of age (30). Our results suggest that NE infants are more likely to have IRAK4 deficiency than controls and may have increased susceptibility to infection. An IRAK4 deficiency may indicate an inability of NE infants to mount a proper immune response. Whether this reduction in IRAK4 is a contributing factor or consequence of $\mathrm{NE}$ remains to be determined in future work.

Infants with NE were LPS-hyporesponsive to NOX2 and CD11b expression and displayed significantly lower expression of IRAK4 compared to neonatal controls. Neutrophils, monocytes, classical monocytes, and non-classical monocytes showed significantly higher mitochondrial mass in NE than controls with LPS. School-age children with a history of NE have significantly altered cytokine responses post-NE compared to controls (31). This dysregulation in immune function may increase susceptibility to infection and correlate with persistent inflammation in childhood (32). Targeting immune function may protect infants with NE from morbidity. In a piglet model, stimulation with LPS $4 \mathrm{~h}$ before hypoxia leads to increased mortality and brain cell death (32). 

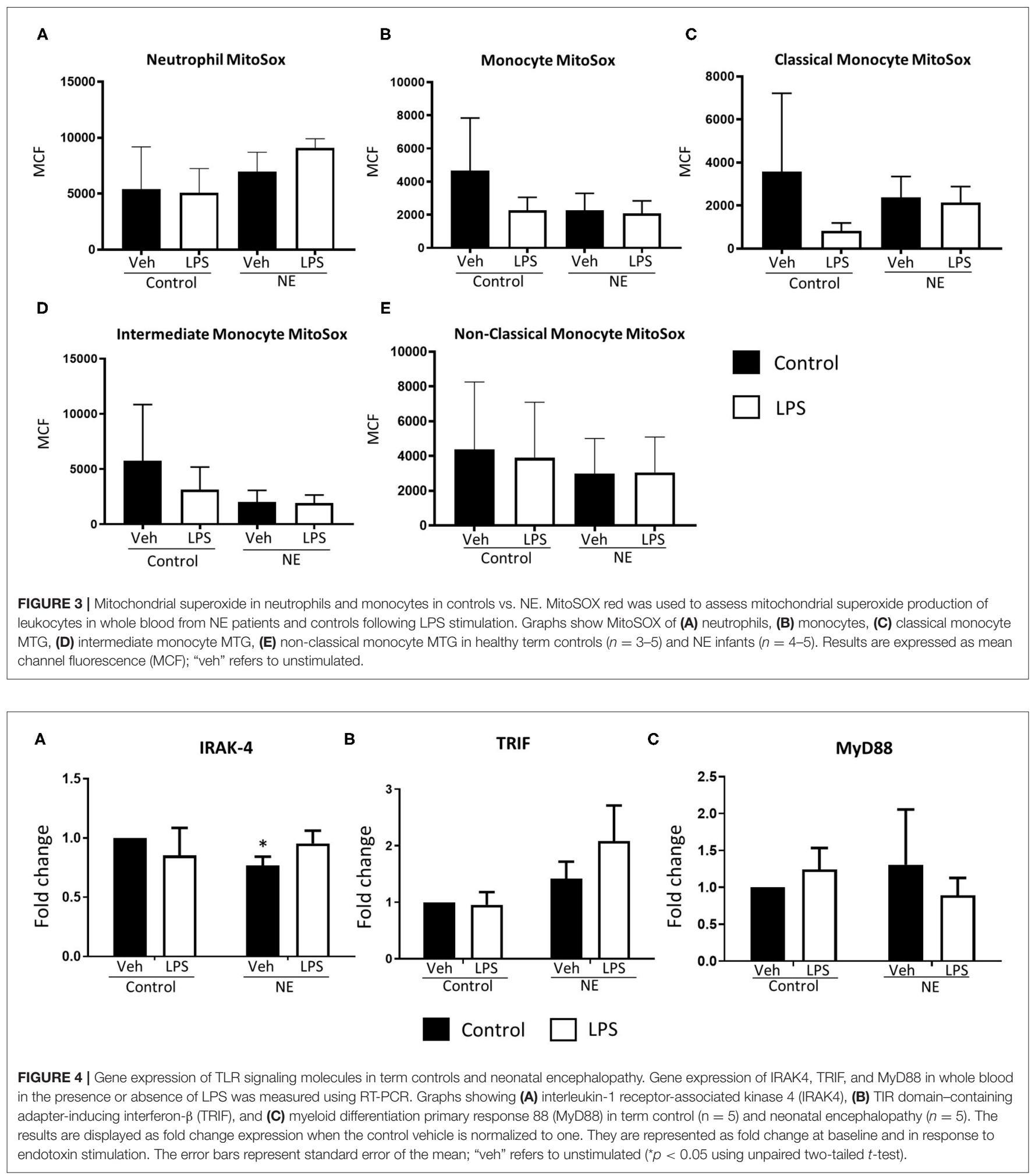

\section{CONCLUSIONS}

$\mathrm{NE}$ infants undergoing $\mathrm{TH}$ have an altered inflammatory response compared to healthy infants, with LPS hyporesponsiveness to CD11b and NOX2, which may indicate a reduced ability to mount an immune response to infection. $\mathrm{NE}$ infants showed a higher mitochondrial mass, which may demonstrate higher metabolic dysfunction and a higher 
production of superoxide, which may be associated with brain injury in NE. Lower IRAK4 gene expression in the NE infants implies that NE infants may have an increased susceptibility to infection, demonstrating that NE infants have an adaptable response to limit hyperinflammation. This article is novel in the use of neonatal blood samples instead of cord blood as a comparator group.

The results are limited by the variability in the timing of phlebotomy, with a median of $2.1( \pm 0.81)$ and two infants having sampling done post-TH. Future directions include comparison of the infant's immunophenotype during and after TH. Future studies are needed to reveal any therapeutic potential of the identified targets, such as CD11b, NOX, and IRAK4.

\section{DATA AVAILABILITY STATEMENT}

The raw data supporting the conclusions of this article will be made available by the authors, without undue reservation.

\section{ETHICS STATEMENT}

The studies involving human participants were reviewed and approved by Dublin Maternity Hospitals. Written informed consent to participate in this study was provided by the participants' legal guardian/next of kin.

\section{AUTHOR CONTRIBUTIONS}

MO'D performed the acquisition, analysis and interpretation of data, carried out statistical analysis, and wrote the manuscript.

\section{REFERENCES}

1. O'hare FM, Watson RW, O'neill A, Blanco A, Donoghue V, Molloy EJ. Persistent systemic monocyte and neutrophil activation in neonatal encephalopathy. J Matern Fetal Neonatal Med. (2016) 29:582-9. doi: 10.3109/14767058.2015.1012060

2. O'hare FM, Watson RW, O'neill A, Segurado R, Sweetman D, Downey P, et al. Serial cytokine alterations and abnormal neuroimaging in newborn infants with encephalopathy. Acta Paediatr. (2017) 106:561-7. doi: 10.1111/apa.13745

3. Shankaran S. Therapeutic hypothermia for neonatal encephalopathy. Curr Treatment Options Neurol. (2012) 14:608-19. doi: 10.1007/s11940-012-0200-y

4. Kuruvilla S, Bustreo F, Kuo T, Mishra CK, Taylor K, Fogstad H, et al. The global strategy for women's, children's and adolescents' health (2016-2030): a roadmap based on evidence and country experience. Bull World Health Organ. (2016) 94:398-400. doi: 10.2471/BLT.16.170431

5. Lee AC, Kozuki N, Blencowe H, Vos T, Bahalim A, Darmstadt GL, et al. Intrapartum-related neonatal encephalopathy incidence and impairment at regional and global levels for 2010 with trends from 1990. Pediatr Res. (2013) 74(Suppl 1):50-72. doi: 10.1038/pr.2013.206

6. Sweetman DU, Onwuneme C, Watson WR, Murphy JF, Molloy EJ. Perinatal asphyxia and erythropoietin and VEGF: serial serum and cerebrospinal fluid responses. Neonatology. (2017) 111:253-9. doi: 10.1159/000448702

7. Liu HD, Li W, Chen ZR, Hu YC, Zhang DD, Shen W, et al. Expression of the NLRP3 inflammasome in cerebral cortex after traumatic brain injury in a rat model. Neurochem Res. (2013) 38:2072-83. doi: 10.1007/s11064-013-1115-z

8. Piras V, Selvarajoo K. Beyond MyD88 and TRIF pathways in toll-like receptor signaling. (2014) 5:70. doi: 10.3389/fimmu.2014.00070
EJM conceived the project, analysis and co-wrote the manuscript, and supervised the experiments. LK, EM, AM, CV, AE-K, JMi, $\mathrm{JMu}$, and $\mathrm{FH}$ assisted in data acquisition, writing, and revision of the manuscript. TH, MN, AB, and GC scored each MRI brain as per Barkovich scoring and that result is now included in the paper post reviewers request. All authors contributed to the article and approved the submitted version.

\section{FUNDING}

This research was funded by the National Children's Research Centre, Dublin and Health Research Board Ireland.

\section{ACKNOWLEDGMENTS}

We wish to thank all the nursing and medical staff in the neonatal units in the Rotunda, National Maternity and Coombe Women's and Infant's University Hospitals and especially the infants and parents who generously agreed to be involved in this project.

\section{SUPPLEMENTARY MATERIAL}

The Supplementary Material for this article can be found online at: https://www.frontiersin.org/articles/10.3389/fped. 2020.598724/full\#supplementary-material

Supplementary Figure 1 | Gating strategy for isolation of granulocytes and monocyte subpopulations. Neutrophils were delineated based on SSC-A and CD66b + positivity. Monocytes were defined based on SSC-A, CD66b- , and subsets based on relative CD14 ${ }^{+} \mathrm{CD} 16^{+}$populations; classical $\left(\mathrm{CD} 14^{+} / \mathrm{CD} 16^{-}\right)$, intermediate $\left(\mathrm{CD} 14^{+} / \mathrm{CD}^{+} 6^{+}\right)$, non-classical $\left(\mathrm{CD} 14 \mathrm{dim} / \mathrm{CD} 16^{+}\right)$.
9. Ravi S, Mitchell T, Kramer P, Chacko B, Darley-Usmar VM. Mitochondria in monocytes and macrophages-implications for translational and basic research. Int J Biochem Cell Biol. (2014) 53:202-7. doi: 10.1016/j.biocel.2014.05.019

10. Levene P, Meyer G. The action of leucocytes on glucose. J Biol Chem. (1912) 361-70. doi: 10.1016/S0021-9258(18)88742-7

11. Sweetman D, Kelly LA, Zareen Z, Nolan B, Murphy J, Boylan G, et al. Coagulation profiles are associated with early clinical outcomes in neonatal encephalopathy. Front Pediatr. (2019) 7:399. doi: 10.3389/fped.2019.00399

12. Sarnat HB, Sarnat MS. Neonatal encephalopathy following fetal distress. Arch Neurol. (1976) 33:696-705. doi: 10.1001/archneur.1976.00500100030012

13. Barkovich AJ, Hajnal BL, Vigneron D, Sola A, Partridge JC, Allen F, et al. Prediction of neuromotor outcome in perinatal asphyxia: evaluation of MR scoring systems. AJNR Am. J. Neuroradiol. (1998) 12:143-9.

14. Leow-Dyke S, Allen C, Denes A, Nilsson O, Maysami S, Bowie AG, et al. Neuronal Toll-like receptor 4 signaling induces brain endothelial activation and neutrophil transmigration in vitro. J Neuroinflamm. (2012) 9:230. doi: 10.1186/1742-2094-9-230

15. Huggard D, Mcgrane F, Lagan N, Roche E, Balfe J, Leahy TR, et al. Altered endotoxin responsiveness in healthy children with down syndrome. BMC Immunol. (2018) 19:31. doi: 10.1186/s12865-018-0270-Z

16. Huggard D, Koay WJ, Kelly L, Mcgrane F, Ryan E, Lagan N, et al. Altered toll-like receptor signalling in children with down syndrome. Media Inflamm. (2019) 2019:4068734. doi: 10.1155/2019/4068734

17. Hickey FB, Corcoran JB, Docherty NG, Griffin B, Bhreathnach U, Furlong F, et al. IHG-1 promotes mitochondrial biogenesis by stabilizing PGC-1alpha. J Am Soc Nephrol. (2011) 22:1475-85. doi: 10.1681/ASN.2010111154 
18. Molloy EJ, O'neill AJ, Grantham-Sloan JJ, Sheridan-Pereira M, Fitzpatrick JM, Webb DW, et al. Neonatal encephalopathy is associated with altered perinatal systemic neutrophil apoptosis. Am J Perinatol. (2007) 24:525-30. doi: 10.1055/s-2007-986678

19. Eliwan HO, Watson WRG, Regan I, Philbin B, O'hare FM, Strickland T, et al. Pediatric intensive care: immunomodulation with activated protein $\mathrm{C}$ ex vivo. (2019) 7:386. doi: 10.3389/fped.2019.00386

20. O'hare FM, Watson W, O'neill A, Grant T, Onwuneme C, Donoghue V, et al. Neutrophil and monocyte toll-like receptor 4, CD11b and reactive oxygen intermediates, and neuroimaging outcomes in preterm infants. Pediatric Res. (2015) 78:82-90. doi: 10.1038/pr.2015.66

21. Hyakkoku K, Hamanaka J, Tsuruma K, Shimazawa M, Tanaka H, Uematsu S, et al. Toll-like receptor 4 (TLR4), but not TLR3 or TLR9, knock-out mice have neuroprotective effects against focal cerebral ischemia. Neuroscience. (2010) 171:258-67. doi: 10.1016/j.neuroscience.2010.08.054

22. Buonocore G, Perrone S, Bracci R. Free radicals and brain damage in the newborn. Biol Neonate. (2001) 79:180-6. doi: 10.1159/000047088

23. Lood C, Blanco LP, Purmalek MM, Carmona-Rivera C, De Ravin SS, Smith $\mathrm{CK}$, et al. Neutrophil extracellular traps enriched in oxidized mitochondrial DNA are interferogenic and contribute to lupus-like disease. Nat Med. (2016) 22:146-53. doi: $10.1038 / \mathrm{nm} .4027$

24. Zhang L, Wang H. Autophagy in traumatic brain injury: a new target for therapeutic intervention. Front Mol Neurosci. (2018) 11:190. doi: 10.3389/fnmol.2018.00190

25. Vorobjeva N, Prikhodko A, Galkin I, Pletjushkina O, Zinovkin R, Sud'ina G, et al. Mitochondrial reactive oxygen species are involved in chemoattractantinduced oxidative burst and degranulation of human neutrophils in vitro. Euro J Cell Biol. (2017) 96:254-65. doi: 10.1016/j.ejcb.2017.03.003

26. Eliwan HO, Watson RW, Aslam S, Regan I, Philbin B, O'hare FM, et al. Neonatal brain injury and systemic inflammation: modulation by activated protein C ex vivo. Clin Exp Immunol. (2015) 179:477-84. doi: 10.1111/cei.1 2453

27. Onwuneme C, Blanco A, O'neill A, Watson B, Molloy EJ. Vitamin D enhances reactive oxygen intermediates production in phagocytic cells in term and preterm infants. Pediatr Res. (2016) 79:654-61. doi: 10.1038/pr. 2015.268

28. Eklind S, Mallard C, Arvidsson P, Hagberg H. Lipopolysaccharide induces both a primary and a secondary phase of sensitization in the developing rat brain. Pediatr Res. (2005) 58:112-6. doi: 10.1203/01.PDR.0000163513.03619.8D

29. Hou Y, Lu X, Zhang Y. IRAK inhibitor protects the intestinal tract of necrotizing enterocolitis by inhibiting the toll-like receptor (TLR) inflammatory signaling pathway in rats. Med. Sci. Monit. (2018) 24:3366-73. doi: 10.12659/MSM.910327

30. Picard C, Von Bernuth H, Ghandil P, Chrabieh M, Levy O, Arkwright PD, et al. Clinical features and outcome of patients with IRAK-4 and MyD88 deficiency. Medicine. (2010) 89:403-25. doi: 10.1097/MD.0b013e3181fd8ec3

31. Zareen Z, Strickland T, Eneaney VM, Kelly LA, Mcdonald D, Sweetman D, et al. Cytokine dysregulation persists in childhood post neonatal encephalopathy. BMC Neurol. (2020) 20:115. doi: 10.1186/s12883-020-01656-w

32. Martinello KA, Meehan C, Avdic-Belltheus A, Lingam I, Ragab S, Hristova M, et al. Acute LPS sensitization and continuous infusion exacerbates hypoxic brain injury in a piglet model of neonatal encephalopathy. Sci Rep. (2019) 9:10184. doi: 10.1038/s41598-019-46488-y

Conflict of Interest: The authors declare that the research was conducted in the absence of any commercial or financial relationships that could be construed as a potential conflict of interest.

Copyright (C) 2021 O’Dea, Kelly, McKenna, Melo, Ni Bhroin, Hurley, Byrne, Colleran, Vavasseur, El-Khuffash, Miletin, Murphy, Hickey and Molloy. This is an open-access article distributed under the terms of the Creative Commons Attribution License (CC $B Y)$. The use, distribution or reproduction in other forums is permitted, provided the original author(s) and the copyright owner(s) are credited and that the original publication in this journal is cited, in accordance with accepted academic practice. No use, distribution or reproduction is permitted which does not comply with these terms. 